\title{
VII. - MESURE DE LA LYSINE LIBRE DU MUSCLE CHEZ LE POULET
}

\author{
M. LARBIER, J. GUILLAUME et C. CALET \\ Station de Recherches avicoles, \\ Centre de Recherches de Tours, I. N.R. A., 37 - Nouzilly
}

RÉSUMÉ

La disponibilité de la lysine des six échantillons de maïs précédemment décrits est évaluée in vivo chez le Poulet à la fois par la mesure du gain de poids et du taux de lysine libre du muscle.

Pour chaque échantillon de maïs, les poulets élevés en cages collectives, reçoivent pendant 8 jours des régimes supplémentés avec des doses croissantes de L-lysine (0- 0,2-0,4 p. 100).

A la suite de ce premier essai, seuls les poulets ayant reçu les régimes les mieux supplémentés en lysine sont conservés et mis en cages individuelles pour mesurer la lysine libre du muscle.

Pour l'ensemble des conditions nutritionnelles, les résultats de croissance ne permettent pas de mettre en évidence de différences significatives entre les six échantillons de maïs. Pourtant, il existe une interaction entre la température de séchage du grain et le taux de lysine du régime: séchés à $80^{\circ} \mathrm{C}$, les maïs présentent une efficacité maximum lorsque le régime n'est pas supplémenté en lysine alors que les maîs séchés à ${ }^{4} 0^{\circ} \mathrm{C}$ permettent les meilleurs gains de poids lorsque Ia lysine est ajoutée au taux le plus fort dans l'aliment.

La teneur du muscle en lysine libre est une mesure extrêmement sensible qui traduit avec précision les dommages causés aux grains par la température et, à un moindre degré, par l'échauffement. Elle est en bonne corrélation avec le test de CARPENTER mais elle ne reflète pas les variations du gain de poids.

\section{INTRODUC'TION}

Depuis longtemps (Faibranks et Mitcheli, i935; Carpenter, I957), on connaît le rôle néfaste de la chaleur et de la conservation sur la valeur biologique des protéines alimentaires. Les acides aminés de ces dernières, et surtout la L-lysine en raison de son groupement $\mathrm{NH}_{2}$ libre, s'associent de manière irréversible à des substances ternaires pour donner des complexes (réaction de MAILLARD, I9I2).

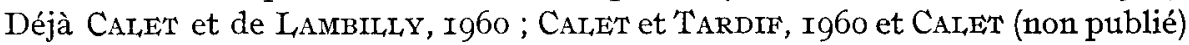
avaient suspecté l'effet des traitements technologiques du maïs sur la disponibilité des acides aminés. Nous nous sommes demandé dans quelle mesure des conditions de préstockage et de séchage, contrôlées de façon rigoureuse, pouvaient altérer la disponibilité de la lysine chez le Poulet. 
On sait depuis les travaux de Zimmerman et ScotT, I965 que le taux des acides aminés libres du plasma du Poulet représente un critère sensible de mesure de l'efficacité des protéines et de la disponibilité de leurs acides aminés. Nous avons perfectionné cette méthode d'une part en appliquant un plan de rationnement précis et d'autre part en nous adressant au muscle puisque ce tissu possède une aptitude toute particulière à concentrer les acides aminés libres (PAWLAK et PION, I968). Dans ces conditions, il existe une relation étroite entre la lysine libre du muscle et la quantité de L-1ysine disponible ingérée par l'animal (LARBIER, GUILIAUME et BLUM, I97I).

Nous avons donc appliqué cette méthode biologique au cas des échantillons de maïs et comparé les résultats à ceux obtenus par les mesures classiques de croissance ou par la méthode chimique de disponibilité de la L-1ysine.

\section{MATÉRIEL ET MÉTHODES}

Dans la première partie de l'expérience, on mesure la réponse de croissance de poussins nourris avec des régimes renfermant $49,5 \mathrm{p}$. Ioo de chaque maîs et supplémentés avec des taux croissants de L-lysine, $\mathrm{HCl}$.

Pour cela nous utilisons des poussins non sexés provenant d'un lot de 324 animaux issus d'un croisement expérimental voisin des croisements commerciaux de type " chair ". Ces poussins

TABLEAU I

Composition du régime préexpérimental (en p. Ioo)

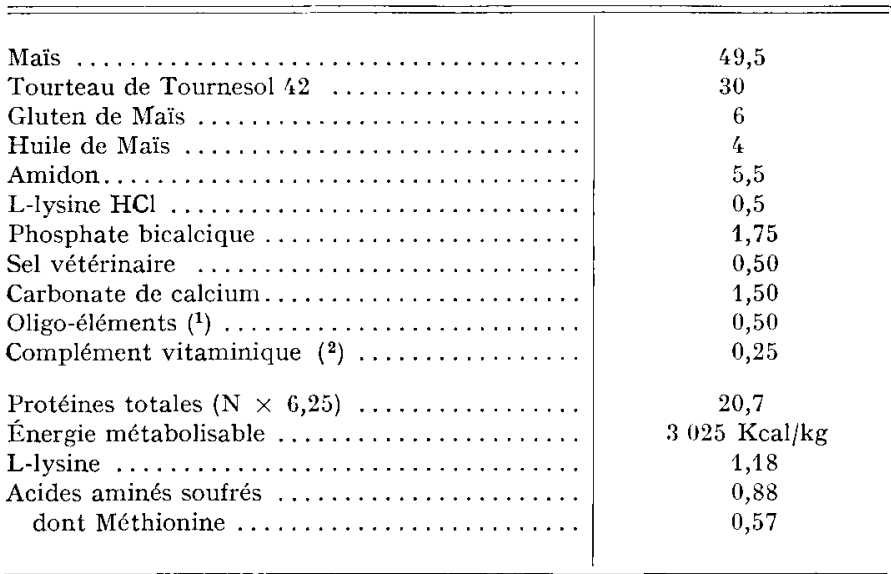

(1) Mélange apportant par $100 \mathrm{~kg}$ d'aliment : sulfate de cuivre $=3,5 \mathrm{~g}$, sulfate de cobalt $=0,5 \mathrm{~g}$, molybdate de sodium $=0,25 \mathrm{~g}$, sulfate de zinc $=75 \mathrm{~g}$, iodure de potassium $=0,75 \mathrm{~g}$, carbonate de calcium $=167 \mathrm{~g}$, Sulfate de maganèse $=100 \mathrm{~g}$, oxyde de magnésie $=50 \mathrm{~g}$, carbonate de magnésie $=40 \mathrm{~g}$, sulfate de fer $=37,5 \mathrm{~g}$.

( $\left.{ }^{2}\right)$ Mélange vitaminique apportant par $100 \mathrm{~kg}$ d'aliment : vitamine $A$ $=2200000 \mathrm{UI}$, vitamine $\mathrm{D}_{3}=280000 \mathrm{UI}, \alpha$-tocophérol $7,5 \mathrm{~g}$, nicotinamide $=1400 \mathrm{mg}$, pantothénate de $\mathrm{Ca}=800 \mathrm{mg}$, vitamine $\mathrm{B}_{12}=0,14 \mathrm{mg}$, riboflavine $=600 \mathrm{mg}$, choline $=15 \mathrm{~g}, \mathrm{BHT}=18 \mathrm{~g}$, avoine q.s.p. $=250 \mathrm{~g}$. 
sont nourris pendant deux semaines avec un régime préexpérimental (tabl. I). On constitue ensuite 36 lots homogènes de 5 animaux. Chaque lot reçoit ad libitum pendant 8 jours l'un des 18 régimes expérimentaux. Ceux-ci dérivent du régime préexpérimental : le maiis ordinaire est remplacé par l'un des 6 mais étudiés et l'apport de L-lysine est de o,-0,2 et o,4 p. roo au lieu de 0,5 p. roo. Ainsi, chaque maïs rentre dans trois régimes qui sont testés sur deux lots de 5 poussins.

Tous ces régimes sont théoriquement complets et équilibrés sauf en ce qui concerne la L-lysine où ils sont au mieux subdéficients.

Les animaux sont pesés au début et à la fin de l'essai. On enregistre également la consommation par cage.

A la suite de cet essai, deux animaux par lot choisis au hasard sont placés en cage individuelle en continuant d'être nourris avec les mêmes régimes. On ne retient pour les résultats que les sujets qui ont reçu les régimes supplémentés de o,4 p. roo de lysine. Pendant cette période, les poulets sont amenés progressivement à ne consommer quotidiennement qu'un seul repas de 2 heures.

Le $7^{\mathrm{e}}$ jour tous les sujets sont sacrifiés après un jeûne de 22 heures. Nous prélevons immédiatement sur chaque poulet une portion de la masse musculaire pectorale gauche et constituons I 8 échantillons en réunissant 2 à 2 les muscles de poulets de même régime.

Les échantillons sont immédiatement plongés dans 1'azote liquide puis maintenus congelés à - I $8^{\circ} \mathrm{C}$ jusqu'à l'analyse. Nous utilisons l'acide sulfosalycylique en solution à $4,5 \mathrm{p}$. Ioo comme agent d'extraction des acides aminés libres.

Le dosage de la lysine libre est effectué au moyen d'un auto-analyseur TECHNICON selon une technique que nous avons mise au point : colonne de $75 \mathrm{~cm}$ contenant de la résine $\mathrm{C}_{2}$, tampon d'élution à $\mathrm{pH}: 4,40$, débit $0,95 \mathrm{ml} / \mathrm{mn}$, température d'élution $37^{\circ} \mathrm{C}$ (LARBIER, non publié).

\section{RÉSULTATS}

\section{I. - Croissance}

Les résultats figurent aux tableaux 2, 3 et 4 . Parmi les trois facteurs étudiés : mode de conservation, température de séchage et supplémentation en L-lysine, seul le dernier a un effet net sur la croissance $(P<0,0 r)$. Les légères différences

\section{TABLEAU 2}

Gain de poids (g)

\begin{tabular}{|c|c|c|c|c|c|}
\hline \multirow{2}{*}{ Type de maïs } & \multicolumn{5}{|c|}{ L-lysine ajoutée en $\%$} \\
\hline & 0 & 0,2 & 0,4 & Moyenne & Moyenne \\
\hline $\mathbf{R}_{80}$ & 43,5 & 66,5 & 71,1 & 60,36 & \\
\hline $\mathrm{R}_{140}$ & 39,9 & 54,5 & 72,6 & 55,71 & \\
\hline $\mathrm{E}_{80}$ & 44,3 & 56,9 & 69,3 & 56,83 & $5750 \quad(\mathrm{E}$ \\
\hline $\mathrm{E}_{140}$ & 39,8 & 53,3 & 81,4 & 58,16 & \\
\hline $\mathrm{F}_{80}$ & 43,2 & 57,9 & 68,1 & 56,4 & $5895(F)$ \\
\hline $\mathrm{F}_{140}$ & 42,1 & 60,6 & 77,6 & 60,11 & \\
\hline Moyennes & 41,95 & 58,32 & 73,52 & & \\
\hline
\end{tabular}


de gain de poids constatées entre les lots d'animaux nourris avec des mais distincts au même taux de supplémentation ne s'avèrent jamais significatives. Il faut cependant souligner l'existence de trois interactions significatives :

TABLEAU 3

Indice de consommation

\begin{tabular}{|c|c|c|c|c|c|}
\hline \multirow{2}{*}{ Maïs } & \multicolumn{5}{|c|}{ L-lysine ajoutée en $\%$} \\
\hline & 0 & 0,2 & 0,4 & Moyenne & Moyenne \\
\hline $\mathrm{R}_{80}$ & 2,28 & 1,77 & 1,59 & 1,88 & \\
\hline $\mathbf{R}_{140}$ & 2,31 & 1,92 & 1,61 & 1,95 & \\
\hline $\mathrm{E}_{80}$ & 2,31 & 1,81 & 1,63 & 1,91 & 192 (F) \\
\hline$E_{140}$ & 2,26 & 1,97 & 1,60 & 1,94 & \\
\hline$F_{80}$ & 2,21 & 1,88 & 1,62 & 1,90 & \\
\hline$F_{140}$ & 2,09 & 1,74 & 1,53 & 1,78 & $2,0<-1=1$ \\
\hline Moyenne & 2,24 & 1,85 & 1,59 & & \\
\hline
\end{tabular}

a) Interaction température $\times$ mode de conservation. La température la plus élevée est préjudiciable aux maïs $R$ et exerce un effet favorable sur les maïs $F$. Par contre, elle ne semble pas altérer le maïs échauffé $\mathrm{E}$.

TABLEAU 4

Consommation moyenne $(\mathrm{g})$

\begin{tabular}{|c|c|c|c|c|c|}
\hline \multirow{2}{*}{ Maïs } & \multicolumn{5}{|c|}{ L-lysine ajoutée en \% } \\
\hline & 0 & 0,2 & 0,4 & Moyenne & Moyenne \\
\hline $\mathrm{R}_{80}$ & 99 & 118 & 113 & 110 & \\
\hline $\mathrm{R}_{\mathbf{1 4 0}}$ & 92 & 105 & 117 & 104,6 & \\
\hline $\mathrm{E}_{\mathbf{8 0}}$ & 102 & 103 & 113 & 106 & \\
\hline$E_{140}$ & 90 & 105 & 130 & 108,3 & \\
\hline$F_{80}$ & 93 & 109 & 112 & 104,6 & \\
\hline$F_{140}$ & 88 & 106 & 119 & 104,3 & \\
\hline Moyenne & 94,0 & 107,6 & 117,3 & & \\
\hline
\end{tabular}


b) Interaction température $\times$ supplémentation en $\mathrm{L}_{\mathrm{L}}$-lysine. Globalement les maïs séchés à $80^{\circ} \mathrm{C}$ donnent les meilleurs gains de poids lorsque le régime est très déficient en L-lysine. Ce sont les résultats opposés que nous observons quand on ajoute $0,4 \mathrm{p}$. Ioo de L-lysine.

Cette interaction est confirmée par l'existence de différences significatives entre les pentes des droites donnant la croissance en fonction du taux de L-lysine ajoutée (fig. I). Tout se passe comme si la supplémentation favorisait davantage les maïs séchés à $140^{\circ} \mathrm{C}$.

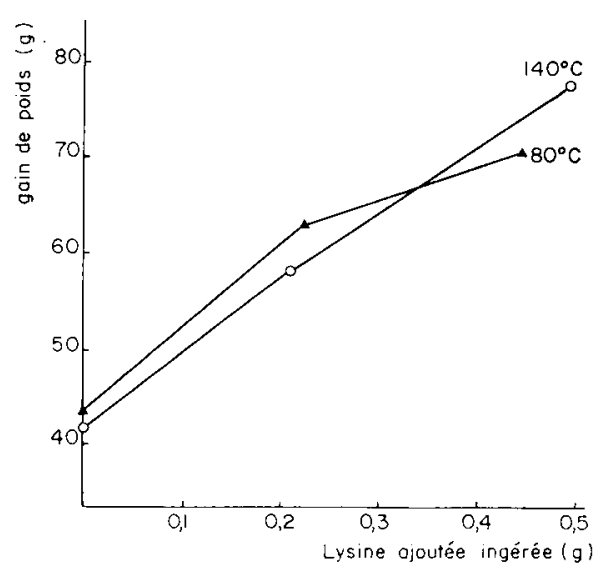

FIG. I. - Influence de la température de séchage du maïs sur la réponse de croissance du Poussin à la supplémentation en lysine

(Ensemble des 3 modes de conservation)

c) Enfin signalons 1'existence d'une interaction du $2^{\mathrm{e}}$ ordre : mode de conservation $\times$ température $\times$ supplémentation dont 1'interprétation est difficile.

On peut donc conclure à l'examen de ces résultats, que les 6 types de mais pris globalement ne sont jamais très différents les uns des autres. L'interaction température $X$ supplémentation peut s'interpréter comme suit : la faible supplémentation en lysine met mieux en lumière la mauvaise disponibilité de cet acide aminé dans les mais traités à $I 40^{\circ} \mathrm{C}$. Une supplémentation plus substantielle $(0,40 \mathrm{p}$. roo) s'accompagnant d'une amélioration de l'appétence des régimes inverse le classement des mais.

L'efficacité alimentaire n'est améliorée de façon nette que par l'apport de L1ysine. Les fluctuations de l'indice de consommation observées par ailleurs résultent surtout des différences de gain de poids ou de consommation.

\section{2. - Mesure de la lysine libre du muscle}

Nous ne considérons la lysine libre du muscle que dans la mesure où le régime est légèrement subdéficient en cet acide aminé. Dans ces conditions (tab1. 5 et 6) nous observons un effet très net des différents facteurs étudiés : mode de conservation et température de séchage. 
a) Mode de conservation.

Les maïs $R$ entraînent les plus fortes teneurs en lysine libre du muscle et les mais $\mathrm{E}$ les plus faibles (24 p. roo de moins).

TABLEAU 5

Lysine libre du muscle en $m g / 100 \mathrm{~g}$ de muscle frais/100 $\mathrm{g}$ d'aliment ingéré L-lysine ajoutée : 0,4 p. 100

\begin{tabular}{l|c|c||c|c||c|c}
\hline & $\mathrm{R}_{\mathbf{8 0}}$ & $\mathrm{R}_{\mathbf{1 4 0}}$ & $\mathrm{E}_{\mathbf{8 0}}$ & $\mathrm{E}_{\mathbf{1 4 0}}$ & $\mathrm{F}_{\mathbf{8 0}}$ & $\mathrm{F}_{\mathbf{1 4 0}}$ \\
\hline & - & & & & \\
\hline Lysine libre. & 9,18 & 7,25 & 8,25 & 4,0 & 7,1 & 6,66 \\
\hline
\end{tabular}

TABLEAU 6

Résultats récapitulatifs exprimés en taux de lysine disponible. et obtenus par différentes méthodes (1)

\begin{tabular}{|c|c|c|c|c|}
\hline \multirow{2}{*}{ Maïs } & \multirow{2}{*}{$\begin{array}{l}\text { Lysine disponible } \\
\text { (CARPENTER) }\left(^{2}\right)\end{array}$} & \multicolumn{2}{|c|}{ Gain de poids } & \multirow{2}{*}{$\begin{array}{c}\begin{array}{c}\text { Lysine libre } \\
\text { du muscle }\end{array} \\
\begin{array}{c}0,4 \% \text { lysine } \\
\text { ajoutée }\end{array}\end{array}$} \\
\hline & & $\begin{array}{c}\text { Sans } \\
\text { supplémentation }\end{array}$ & $\begin{array}{l}0,4 \% \text { lysine } \\
\text { ajoutée }\end{array}$ & \\
\hline $\mathrm{R}_{\mathbf{8 0}}$ & 100 & 100 & 100 & 100 \\
\hline $\mathrm{R}_{140}$ & 88 & 91,7 & 102,1 & 78,5 \\
\hline $\mathrm{E}_{80}$ & 97 & 101,8 & 97,5 & 92 \\
\hline$E_{140}$ & 79 & 91,5 & $11^{4}, 5$ & 44 \\
\hline $\mathrm{F}_{80}$ & 96,5 & 99,3 & 97,2 & 77 \\
\hline $\mathrm{F}_{140}$ & 82 & 96,8 & 109,1 & 72 \\
\hline $\mathrm{R}$ & 94 & 95,9 & 101,1 & 89 \\
\hline $\mathrm{E}$ & 88 & 96,7 & 106,0 & 68 \\
\hline $\mathrm{F}$ & 88 & 98,1 & 103,2 & 75 \\
\hline 80 & 98 & 100,4 & 98,2 & 90 \\
\hline 140 & 83 & 93,3 & 108,6 & 65 \\
\hline
\end{tabular}

(1) Dans tous les cas on a distribué la valeur 100 au maïs $R_{\mathbf{8 0}}$ considéré comme témoin.

(2) Cf. rapport de B. Godon, et L. Petit (V).

b) Température de séchage.

Quel que soit le mode de conservation, le séchage à $140^{\circ} \mathrm{C}$ semble préjudiciable au dépôt de la lysine sous forme libre dans le muscle. Ainsi pour une même quantité d'aliment ingérée (fig. 2) la teneur en lysine libre du muscle est toujours plus élevée quand les animaux sont nourris avec des maïs séchés à $80^{\circ} \mathrm{C}$. Nous avons ainsi une très nette illustration de 1'effet particulièrement défavorable de la température la plus élevée $\left(\mathrm{I}_{4} 0^{\circ} \mathrm{C}\right)$ sur la disponibilité de la lysine. 


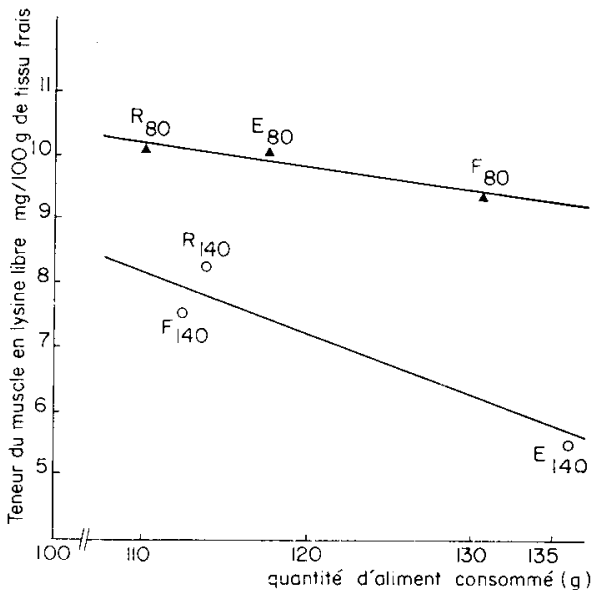

FIG. 2. - Relation entre quantité d'aliment ingérée et teneur en lysine libre du muscle de poulet nourri des différents échantillons de mä̈s

Bien que l'on ne puisse pas vérifier statistiquement l'existence d'une interaction entre ces facteurs, le traitement $\mathrm{F}$ semble être le plus défavorable quand la température de séchage est de $80^{\circ} \mathrm{C}$ alors que pour $\mathrm{I}_{40^{\circ} \mathrm{C}} \mathrm{c}^{\prime}$ est $\mathrm{E}$ qui donne la plus faible disponibilité.

\section{DISCUSSION}

Nous avons comparé nos résultats concernant le gain de poids, l'efficacité alimentaire et la teneur en 1ysine libre du muscle d'une part au taux de lysine disponible mesuré sur ces mêmes maïs par Godon et PE'IIT. (V) ; d'autre part dans le tableau 6 nous avons présenté les résultats en pourcentage du lot $R_{80}$.

Io La teneur en lysine libre du muscle est en corrélation positive et élevée avec le taux de lysine disponible mesuré in vitro $(r=0,87, \mathrm{P}=0,05)$. Le test biologique que nous proposons est toutefois plus sensible. Exprimées en terme de disponibilité, les valeurs obtenues par cette méthode se situent dans une gamme plus étalée que ne le sont les résultats in vitro comme l'indique l'équation de régression :

$$
\begin{aligned}
& y=\mathrm{I}, 93 x-97,24 \text { où } \\
& y=\text { teneur en lysine libre du muscle }\left(y=\text { Ioo pour } \mathrm{R}_{80}\right) \\
& \text { et } \quad x=\text { taux de 1ysine disponible mesuré in vitro. }
\end{aligned}
$$

$2^{\circ}$ La teneur en lysine libre du muscle rend compte d'une manière très nette des dommages subis par les protides du maïs sous l'effet des traitements puisque sa valeur peut être réduite de moitié. Une fois de plus la sensibilité de cette mesure est mise en lumière.

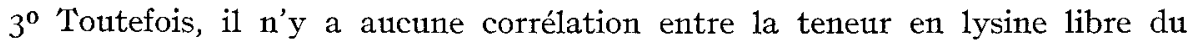
muscle et le gain de poids ou l'efficacité alimentaire chez des sujets recevant un régime subdéficient (supplément de $0,4 \mathrm{p}$. Ioo de lysine). Les coefficients de corrélation, non significatifs, sont respectivement de 0,06 et - o, I7. 
Enn revanche, lorsque les régimes sont très déficients en 1ysine (sans apport de supplément) le gain de poids des poulets rend compte - à un degré plus faible que les tests de laboratoire — de l'effet de la température sur l'efficacité des grains. C'est néanmoins le seul cas où la lysine disponible et le gain de poids vont dans le même sens.

\section{CONCLUSION}

L'étude de la lysine libre du muscle de Poulet dans des conditions d'alimentation bien particulières permet d'évaluer la teneur du maïs en 1ysine disponible. Nos résultats sont en bon accord avec ceux obtenus par l'analyse chimique (méthode de CARPENTER). Ils démontrent clairement les effets néfastes de la conservation par ventilation d'air refroidi, de l'échauffement, du séchage à I $40^{\circ} \mathrm{C}$ et surtout de la combinaison échauffement — séchage à $\mathrm{I}_{40}{ }^{\circ} \mathrm{C}$. $\mathrm{La}$ mesure que nous proposons constitue un critère encore plus sensible de la disponibilité de la lysine.

Les tests de croissance en revanche ne fournissent qu'une réponse globale des effets des traitements non spécifiques de la lysine disponible même quand celle-ci est le facteur limitant primaire des protéines.

\section{SUMMARY}

\section{VII. - MUSCLE IEVEI, OF FREE IYSINE IN THE CHICK}

The availability of the lysine in the six samples of maize previously described was evaluated in vivo in the chick using body weight gain and muscle level of free lysine.

The chicks, reared in collective cages, were fed diets supplemented with $0,0.2$ and $0.4 \mathrm{p}$. roo L-lysine for each sample of maize. At the end of this trial only birds fed the diets supplemented with 0.4 p. too lysine were kept and reared in individual cages for measurement of muscle free lysine.

Growth trial results did not show any general difference among the samples of maize. However an interaction between drying temperature and dietary lysine level appeared : without lysine supplementation maizes were most efficient when dried at $80^{\circ} \mathrm{C}$ while with 0.4 L-lysine supplementation growth was maximum when maizes had been dried at $140^{\circ} \mathrm{C}$.

The muscle level of free lysine was a very sensitive measurement that indicated with accuracy the damages occasionned to maize by high temperature or, at a smaller degree, by heating. It was in good correlation with available lysine according to CARPENTER but it did not indicate the weight gain variations.

\section{RÉFÉRENCES BIBLIOGRAPHIQUES}

Calet C., de lambilly H., r960. Étude de la valeur alimentaire du maïs grain séché artificiellement pour le poussin en croissance. I. Influence du mode de séchage sur la disponibilité des acides aminés. Ann. Zootech., 9, I8I-I84.

CAlet C., TARDif H., Ig6o. Étude de la valeur alimentaire du maïs grain séché artificiellement pour le poussin en croissance. II. Influence de la durée qui sépare la récolte du séchage. Ann. Zootech., 9, 349-354.

Carpenter J. K., I957. The chemical and biological assay of lysine in foods, $4^{\mathrm{e}}$ Congrès intern. de Nutrition, Résumés des communications, I54-I55, Paris. 
Faibranks B. W., Mrtchell H. H., I935. The nutritive value of skim milk powders, with special reference to the sensivity of milk proteins to heat. J. Agri. Res., 51, Iro7-II2I.

Larbier M., Guillaume J., Blum J. C., 1971. Muscle levels of free lysine and methionine in chicks fed a single daily meal. Nutr. Rep. Int. 3, 273-276.

Maillard L. C., Igr2. Action des acides aminés sur les sucres. Formations des mélanoïdines par voie méthodique. C. R. Acad. Sci., 154, 66-68.

Pawlak M., Pion R., I968. Influence de la supplémentation des protéines de blé par des doses croissantes de lysine sur la teneur en acides aminés libres du sang et du muscle du rat en croissance. $A n n$. Biol. anim. Bioch. Biophys., 8, 517-530.

Zimmerman R. A., ScotT H. M., I965. Interrelationship of plasma amino acid levels and weight gain in the chick as infuenced by suboptimal and superoptimal dietary concentration of single amino acid. J. Nutr., 87, 13-18. 